\title{
CENTRALITY ANALYSIS FOR MODIFIED LATTICES
}

\author{
MARTIN PATON* ${ }^{*}$ KEREM AKARTUNALI ${ }^{\dagger}$, AND DESMOND J. HIGHAM ${ }^{\ddagger}$
}

\begin{abstract}
We derive new, exact expressions for network centrality vectors associated with classical Watts-Strogatz style "ring plus shortcut" networks. We also derive easy-to-interpret approximations that are highly accurate in the large network limit. The analysis helps us to understand the role of the Katz parameter and the PageRank parameter, to compare linear system and eigenvalue based centrality measures, and to predict the behavior of centrality measures on more complicated networks.
\end{abstract}

Keywords: circulant, Katz, network, PageRank, ring, shortcut, spectrum.

AMS Subject Classification: 05C82, 05C50

1. Background and Notation. Algorithms that quantify the importance of nodes in a network are proving to be extremely useful [12, 25, 29]. They allow us to understand hierarchies, discover critical components, and identify targets for deeper investigation. Many of the key ideas behind network centrality measures arose out of the social sciences, where researchers were interested in understanding structural attributes of human interaction networks [13]. The ability to determine who or what is important is also valuable in many application areas, including healthcare, security, advertising, publishing and politics [2, 15, 21, 23].

A key issue, and perhaps a reason for the continued development of new ideas in the field, is that there is no universally-agreed definition (or set of definitions) for importance, and hence no gold-standards for judging centrality measures. So, issues such as validating implementations, understanding the role of algorithm parameters, and comparing centrality measures can only be partially addressed, typically by using real world data sets where some proxy for importance is available, leading to conclusions that are (a) empirically based and (b) problem-set dependent. In this work, we contribute to the field by showing that there is a synthetic but widely studied "small world" type network for which we can analytically characterize and compare four well-known centrality measures - degree, Katz, eigenvector and PageRank. In particular, we can quantify how the node centralities change as the Katz parameter moves from zero (degree centrality) to its upper limit (eigenvector centrality). Moreover, we show how the same techniques allow us to characterize fully these measures on more complex networks where the performance can depend strongly on the choice of Katz or PageRank parameter.

We consider simple unweighted, directed graphs with $N$ nodes. We let $A \in \mathbb{R}^{N \times N}$ denote the adjacency matrix, so that $a_{i j}=1$ if there is an edge from node $i$ to node $j$ and $a_{i j}=0$ otherwise. We also let $\rho(A)$ denote the spectral radius of $A$, and let $1 \in \mathbb{R}^{N}$ denote the vector of ones.

Section 2 gives a brief review of Katz, eigenvector and PageRank centrality measures. Section 3 summarizes relevant work on Watts-Strogatz style small world networks and Section 4 sets up the matrix formulation for our asymptotic analysis. Katz

*Department of Mathematics and Statistics, University of Strathclyde, Glasgow G1 1XH, UK (martin.paton@strath.ac.uk). Supported by University of Strathclyde Strategic Technology Partnership with CAPITA.

${ }^{\dagger}$ Department of Management Science, University of Strathclyde, Glasgow G4 0GE, UK (kerem.akartunali@strath.ac.uk). Corresponding author.

${ }^{\ddagger}$ Department of Mathematics and Statistics, University of Strathclyde, Glasgow G1 1XH, UK (d.j.higham@strath.ac.uk). Supported by EPSRC/RCUK Established Career Fellowship EP/M00158X/1 and a Royal Society/Wolfson Research Merit Award. 
centrality and eigenvector centrality are analysed in Sections 5 and 6, respectively. In Section 7 we apply the same style of analysis to a more general network and find an analytical expression for the parameter cutoff where the ranking from out-degree gives way to that from eigenvector centrality. We also experiment on an extended lattice structure with multiple shortcuts.

Section 8 gives analysis for PageRank centrality - here the exact solution is found to have a more complicated, non-monotonic, form. We finish in Section 9 with a brief discussion.

2. Network Centrality Measures. In this section we assume that the network is strongly connected, so that $A$ is irreducible. This property holds for the network class that we study in subsequent sections.

Given $A$, a centrality measure assigns a value $x_{i}>0$ to each node $i$, with a larger value indicating a greater level of importance. Typically, it is the ranking of the centrality values that matters - we only care whether one node is more or less important than another, so the vector $x \in \mathbb{R}^{N}$ is equivalent to $\beta x+\gamma \mathbf{1}$ for any $\beta, \gamma>0$. We summarize here the concepts of Katz, eigenvector and degree centrality, refering to $[1,6,12,25,29]$ for historical details and discussions of implementation issues. We also consider the PageRank algorithm [14, 20, 28] which has a different feel; summarizing incoming, rather than outgoing, information, but also assigns a positive real value to each node. Our overall aim is to study, in a specific setting, how changes to the network affect centrality. We note that related questions have been addressed in other contexts; see, for example, $[5,11]$.

Katz centrality [19] defines $x$ via the linear system

$$
(I-\alpha A) x=\mathbf{1}
$$

where $\alpha \in(0,1 / \rho(A))$ is a free parameter. Several authors have suggested particular choices for $\alpha$; see [1] and the references therein. This measure can be motivated by expanding the resolvent $(I-\alpha A)^{-1}$ to give

$$
x=\left(I+\alpha A+\alpha^{2} A^{2}+\alpha^{3} A^{3}+\cdots\right) \mathbf{1} .
$$

Noting that $\left(A^{k}\right)_{i j}$ counts the number of distinct walks of length $k$ from $i$ to $j$, we see that $x_{i}$ is a weighted sum of the number of walks from node $i$ to all other nodes, where the count for walks of length $k$ is scaled by $\alpha^{k}$. So $x_{i}$ is a measure of how well node $i$ can send information around the network, with more weight given to shorter traversals.

In the limit as $\alpha \rightarrow 0$ from above the ranking given by (1) coincides with the ranking from degree centrality, which arises from taking $x_{i}$ to be the out-degree of node $i$; that is,

$$
x_{i}=\operatorname{outdeg}_{i}, \quad \text { where outdeg }=A \mathbf{1} .
$$

This makes sense intuitively, since accounting only for the shortest possible walks - of length one - is equivalent to computing the out-degree.

Eigenvector centrality can be motivated recursively, with a node being important if it has links to important nodes. This leads to the expression

$$
x_{i} \propto \sum_{j=1}^{N} a_{i j} x_{j} .
$$


Letting $1 / \lambda$ denote the constant of proportionality, we may write

$$
A x=\lambda x,
$$

showing that $x$ must be an eigenvector of $A$ corresponding to eigenvalue $\lambda$. Requiring $x_{i}>0$ for all $i$ forces $\lambda$ and $x$ to be the Perron-Frobenius eigenvalue and eigenvector, respectively.

In the limit as $\alpha$ tends to $1 / \rho(A)$ from below, the Katz centrality vector in (1) gives the same results as the eigenvector centrality vector; see, for example, [4].

The PageRank algorithm, originally proposed in [28], can be motivated from several different perspectives. For example, keeping in mind the context of web pages, we could alter (3) by arguing that the importance of a node is dependent on the importance of the nodes that point to it. If $a_{i j}=1$ indicates a hyperlink from node $i$ to node $j$, then we can set up an iteration where each node is given a convex combination of a basal score and a normalized sum of the scores of its followers, so that

$$
x_{i}^{[k+1]}=1-d+d \sum_{j=1}^{N} \frac{a_{j i}}{\operatorname{outdeg}_{j}} x_{j}^{[k]} .
$$

Here, scaling by outdeg ${ }_{j}$ ensures that every node has the same opportunity to distribute its influence across its neighbours. The parameter $0<d<1$ controls the relative weight given to this redistribution. Taking the limit $k \rightarrow \infty$, we define the PageRank vector as the solution of

$$
\left(I-d A^{T} D^{-1}\right) x=(1-d) \mathbf{1},
$$

where $D=\operatorname{diag}\left(\operatorname{outdeg}_{j}\right)$. We refer to $[14,20]$ for further details.

3. Small World Networks. The seminal work of Watts and Strogatz [30] introduced a class of random graphs characterised by having many "local" links and a few "long range" links. Those authors showed, via computational experiments, that such a model can reproduce clustering and pathlength properties that have been observed in real-world complex networks. A key idea in [30] was to add randomness to a regular lattice. Starting from an undirected periodic ring with fixed-range nearest-neighbor connections, the authors introduced a rewiring procedure - each node in the lattice was examined in turn, and, for each of its undirected links, with small independent probability the end point of that link was replaced by another node chosen uniformly at random. In terms of developing analytical results to back up the observations in [30], the rewiring process presents difficulties; for example, a node may become isolated with nonzero probability. For this reason, subsequent research focused on a slight variation where existing edges are not altered, but new edges, termed shortcuts, are added between randomly selected nodes in the network. For example, focusing on the $N \rightarrow \infty$ limit, [24, 26, 27] develop heuristic approximations and [3] gives rigorous results. Markov chain versions with hitting time as a proxy for pathlength were also studied rigorously in $[10,16,17,31]$. Of particular relevance to our study is the reference [22], which analysed the effect of shortcuts on the underlying matrix spectrum from a linear algebra viewpoint.

In this work we will therefore use the shortcut concept, rather than rewiring. The details are described in the next section. We also note that in the case where each undirected edge in the underlying periodic ring is regarded as an independent pair of directed edges, so that a rewired edge produces a directed link, the study of Katz and 
eigenvalue centralities is not interesting. This can be seen from the following simple lemma.

Lemma 1. Suppose that all nodes in a network have the same out-degree. Then all nodes have the same Katz centrality measure. Similarly, assuming strong connectivity, all nodes have the same eigenvector centrality measure.

Proof. We have

$$
A \mathbf{1}=\operatorname{od} \mathbf{1},
$$

where od $\equiv$ outdeg $_{i}$ denotes the common out-degree. Hence $\mathbf{1}$ is the Perron-Frobenius eigenvector and $\rho(A)=$ od. Any Katz parameter $0<\alpha<1 /$ od is valid, and we see from (6) that $x=\mathbf{1} /(1-\alpha$ od) solves the Katz system (1).

4. Matrix Modification. Let $C \in \mathbb{R}^{N \times N}$ be the adjacency matrix for the periodic nearest neighbour ring; so in the $N=6$ case,

$$
C=\left[\begin{array}{llllll}
1 & 1 & & & & 1 \\
& 1 & & 1 & & \\
& & 1 & & 1 & \\
& & & 1 & & 1 \\
1 & & & & 1 &
\end{array}\right]
$$

We note that $C$ is symmetric and circulant, and standard theory shows that the Perron-Frobenius eigenvalue is $\rho(C)=2$ with corresponding eigenvector $x=\mathbf{1}$ [18, page 100], which, from Lemma 1, also solves the Katz system (1), up to a multiplicative factor, for any $0<\alpha<1 / \rho(C)$. It is, of course, intuitively reasonable that all nodes in this network should be assigned the same centrality value in (1), (2) and (4).

Next we add a single directed shortcut. Without loss of generality we give the shortcut to node 1 and let $L$ be the index of the target node. So our adjacency matrix $A$ in (1) has the form $A=C+E$, where the rank one matrix $E$ is zero except for $E(1, L)=1$. Liu, Strang and Ott [22] describe this as a modification of $C$, to emphasize that we have an $O(1)$ change in a matrix entry, rather than the type of small change studied in classical matrix perturbation theory. These authors studied the eigenvector associated with the dominant eigenvalue of $A$, and related matrices, and constructed accurate approximations to this vector. Further work concerning the eigenvalues arising from general modifications to structured matrices has appeared in, for example, $[7,8,9]$.

Our work is strongly motivated by [22] but differs from it in three respects.

- Rather than deriving small residual approximations and then using stability arguments to bound the forward error, we construct exact solutions that can be expanded asymptotically. This more direct route leads to shorter proofs and sharper bounds.

- We consider Katz and PageRank centrality (as well as the eigenvalue problem).

- We interpret the results from a network science perspective and show how they can give new insights about behavior on more complicated networks.

For convenience, we let $p(i)$ for any $1 \leq i \leq N$ denote the periodic distance from node $i$ to node 1 , that is,

$$
p(i)=\min (i-1, N-i+1) .
$$



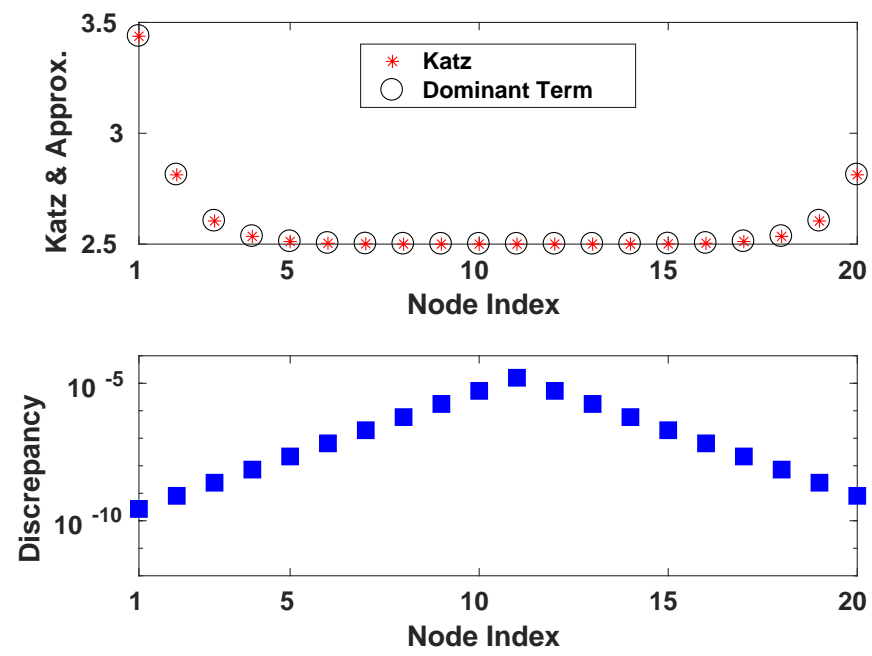

FIG. 1. Upper picture: asterisks show components of Katz vector from (1) and circles show the approximation $b+h_{1} t_{1}^{p(i)}$ from (12). Here $b=1 /(1-2 \alpha), t_{1}$ is defined in (11) and $h_{1}$ was found by solving (20). Lower picture: the discrepancy $x_{i}-b-h_{1} t_{1}^{p(i)}$ on a log scale. From Theorem 1, this quantity has the form $h_{2} t_{2}^{p(i)}$, and hence grows geometrically away from the shortcut node. However, it is uniformly $O\left(t_{1}^{N / 2}\right)$ for a fixed $0<t_{1}<1$, and hence rapidly becomes negligible as the network size $N$ increases.

We can assume without loss of generality that the receiving node $L$ is not beyond the half way, or "six o'clock", position on the ring. We are interested in large networks with long-range shortcuts. So, letting $\lfloor\cdot \mid$ denote the integer part, for some fixed proportion $0<\theta \leq 1$ we set

$$
L= \begin{cases}\lfloor\theta(N / 2+1)\rfloor & \text { when } N \text { is even, } \\ \lfloor\theta(N+1) / 2\rfloor & \text { when } N \text { is odd. }\end{cases}
$$

We note that $L \rightarrow \infty$ as $N \rightarrow \infty$.

5. Katz Centrality. In the upper picture of Figure 1, the asterisks show Katz centrality values for a network with adjacency matrix $A=C+E$, that is, components of $x$ from (1). We chose a small network size in order to make the key effects visible. More precisely, we used an $N=20$ node ring with a shortcut from node 1 to node $L=8$, for $\alpha=0.3$. Because node 1 owns the extra, long-range edge, it attains the highest centrality score, at around 3.5. The most distant node, periodically, node 11, is deemed the least central. Insight from [22], or from eyeballing the solution, suggests that components of $x_{i}$, when suitably shifted, might be varying geometrically as the index $i$ moves periodically around the ring; that is $x_{i}=b+h t^{p(i)}$ for some constants $b, h>0$ and $0<t<1$. Fitting an ansatz of this form leads us to the circles in the upper picture of Figure 1. The agreement is close - below $2 \times 10^{-5}$ in Euclidean norm.

The lower picture in Figure 1 shows, on a log scale, the discrepancy between those asterisks (true solution) and circles (geometric decay ansatz). We see a very small contribution that, in contrast to the overall solution, grows geometrically as we move periodically away from node 1 .

We now state a theorem that explains Figure 1. The theorem concerns the limit 
$N \rightarrow \infty$ with a fixed Katz parameter $0<\alpha<1 / 2$. This upper limit for $\alpha$ is chosen because, as proved in section 10, $\rho(A)$ tends to 2 from above as $N \rightarrow \infty$.

TheOREM 1. For the undirected ring plus directed shortcut network with adjacency matrix $A=C+E$, for sufficiently large $N$ the unique solution of the Katz system (1) has the form

$$
x_{i}=b+h_{1} t_{1}^{p(i)}+h_{2} t_{2}^{p(i)} .
$$

Here, $b, t_{1}, t_{2}, h_{1}, h_{2}$ are constants, i.e., independent of $i$, and $b, t_{1}, t_{2}$ are also independent of $N$. In particular, $h_{1}>0, h_{2}>0, b=1 /(1-2 \alpha)$ and $t_{1}, t_{2}$ are the roots of the palindromic quadratic $\alpha t^{2}-t+\alpha$, so that

$$
t_{1}=\frac{1-\sqrt{1-4 \alpha^{2}}}{2 \alpha}, \quad t_{2}=\frac{1+\sqrt{1-4 \alpha^{2}}}{2 \alpha},
$$

with $t_{2}=1 / t_{1}$ and $0<t_{1}<1<t_{2}$. Moreover, the final term in (10) is exponentially small asymptotically, in the sense that for all $1 \leq i \leq N$,

$$
x_{i}=b+h_{1} t_{1}^{p(i)}+O\left(t_{1}^{N / 2}\right),
$$

with $h_{1}=O(1)$.

Proof. Because the quadratic is palindromic, $t_{1}$ and $t_{2}$ in (11) must satisfy $t_{1} t_{2}=$ 1. It is clear that these roots are real, with $0<t_{1}<1<t_{2}$, and we note the further useful facts

$$
1-2 \alpha t_{1}=\sqrt{1-4 \alpha^{2}}>0, \quad 1-2 \alpha t_{2}=-\sqrt{1-4 \alpha^{2}}<0
$$

and

$$
\frac{t_{1}-2 \alpha}{t_{2}-2 \alpha}=-t_{1}^{2} .
$$

Consider the case where the number of nodes, $N$, is even. We begin with the ansatz

$$
x_{i}=b+h t^{p(i)} .
$$

The Katz system (1) essentially reduces to three scalar equations. At a general node $k$, corresponding to the $k$ th row of the linear system, for $k \neq 1$ and $k \neq N / 2+1$ we have

$$
-\alpha x_{k-1}+x_{k}-\alpha x_{k+1}=1 .
$$

Inserting (15), this becomes

$$
b(1-2 \alpha)+h t^{p(k)}[1-\alpha t-\alpha / t]=1 .
$$

We can satisfy this equation independently of $k$ by setting $b=1 /(1-2 \alpha)$ and choosing $t$ to be either root of the quadratic $\alpha t^{2}-t+\alpha$. By linearity of (16), we may extend (15) to include a linear combination involving both roots, so our ansatz becomes

$$
x_{k}=b+h_{1} t_{1}^{p(k)}+h_{2} t_{2}^{p(k)} .
$$


The two remaining equations to satisfy from (1) arise at nodes 1 and $N / 2+1$, where we require

$$
-\alpha x_{N}+x_{1}-\alpha x_{2}-\alpha x_{L}=1
$$

and

$$
-\alpha x_{N / 2}+x_{N / 2+1}-\alpha x_{N / 2+2}=1,
$$

respectively. Inserting (17) into (18) and (19), using $b(1-2 \alpha)=1$, we have the linear system

$$
\left[\begin{array}{cc}
1-2 \alpha t_{1}-\alpha t_{1}^{L-1} & 1-2 \alpha t_{2}-\alpha t_{2}^{L-1} \\
t_{1}^{N / 2-1}\left(t_{1}-2 \alpha\right) & t_{2}^{N / 2-1}\left(t_{2}-2 \alpha\right)
\end{array}\right]\left[\begin{array}{c}
h_{1} \\
h_{2}
\end{array}\right]=\left[\begin{array}{c}
b \alpha \\
0
\end{array}\right]
$$

To prove that a solution of the form (17) exists, we now show that this system is nonsingular. The determinant may be written

$$
\left(1-2 \alpha t_{1}-\alpha t_{1}^{L-1}\right) t_{2}^{N / 2}\left(1-2 \alpha t_{1}\right)-\left(1-2 \alpha t_{2}-\alpha t_{2}^{L-1}\right) t_{1}^{N / 2}\left(1-2 \alpha t_{2}\right) .
$$

Recalling that $0<t_{1}<1<t_{2}$, we see from (13) that, as $N \rightarrow \infty$ and hence $L \rightarrow \infty$, the first term in (21) becomes, to leading order,

$$
\left(1-2 \alpha t_{1}\right)^{2} t_{2}^{N / 2} \rightarrow \infty .
$$

The second term in (21) becomes, to leading order,

$$
\alpha t_{2}^{L-1} t_{1}^{N / 2}\left(1-2 \alpha t_{2}\right)=\alpha t_{1}^{N / 2-L}\left(t_{1}-2 \alpha\right) .
$$

Recall that $L=\lfloor\theta(N / 2+1)\rfloor$, so

$$
N / 2-L>N / 2-\theta(N / 2+1)=(1-\theta) N / 2-\theta .
$$

Hence, as $N \rightarrow \infty$, term $\alpha t_{1}^{N / 2-L}\left(t_{1}-2 \alpha\right)$ in (22) is bounded for $\theta=1$, and tends to zero for $\theta<1$. So the first term in (21) dominates, and the determinant is bounded away from zero for large $N$.

We have shown that for sufficiently large $N$ the unique solution of the Katz system (1) has the form (17). We now follow up by showing that, in the exact solution (17), the growing term $h_{2} t_{2}^{p(i)}$ is negligible for large $N$.

From the second equation in (20), we have, using (14),

$$
h_{2}=h_{1} t_{1}^{N} .
$$

Hence, in the first equation of (20),

$$
h_{1}\left[1-2 \alpha t_{1}-\alpha t_{1}^{L-1}-\alpha t_{1}^{N-L+1}-2 \alpha t_{1}^{N-1}+t_{1}^{N}\right]=b \alpha .
$$

We see that $h_{1}=O(1)$. Also, using (13), we deduce that $h_{1}>0$ for large $N$. It then follows from (23) that $h_{2}>0$ and $h_{2}=O\left(t_{1}^{N}\right)$. Since the term $h_{2} t_{2}^{p(k)}$ is largest when $k=N / 2+1$, we also see that

$$
x_{i}=b+h_{1} t_{1}^{p(i)}+O\left(t_{1}^{N / 2}\right) .
$$

The case where $N$ is odd can be handled in a similar way. 

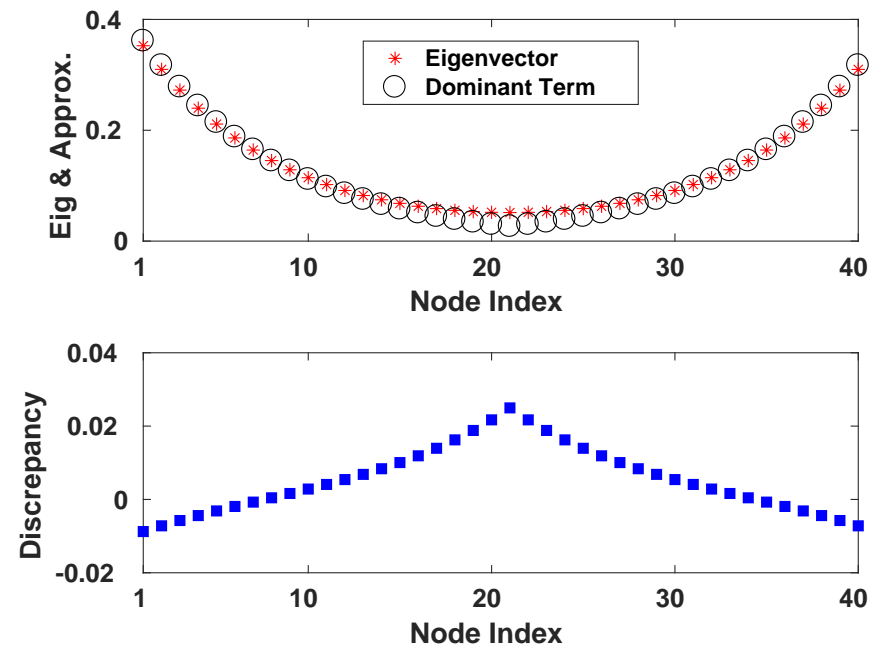

FIG. 2. Upper picture: asterisks show components of the Perron-Frobenius vector and circles show the approximation $s_{1}^{p(i)}$ from (24), with $s_{1}$ defined in (25). Both vectors are normalized to have unit Euclidean norm. Lower picture: the discrepancy. From Theorem 2, $x_{i}-s_{1}^{p(i)}$ has the form $s_{1}^{N-p(i)}$, and hence grows geometrically away from the shortcut node.

6. Eigenvector Centrality. This section looks at eigenvector centrality for the matrix $C+E$. We note that $\rho(C+E) \leq\|C+E\|_{\infty}=3$. Also, it is true in general that adding an edge to a strongly connected network strictly increases the spectral radius; see [18, Problem 8.4P14]. So $2<\rho(C+E)$. From the network centrality perspective, we are concerned mainly with the structure of the PerronFrobenius eigenvector. However, for completeness, in Section 10 we establish a tight bound on the corresponding eigenvalue.

The asterisks in the upper picture of Figure 2 show the components of the PerronFrobenius vector for $A=C+E$ in the case where $N=40$ and $L=12$. As in the Katz case seen in Figure 1, there is evidence of periodic geometric decay. The circles in the picture show an ansatz of the form $x_{i}=s_{1}^{p(i)}$ for $0<s_{1}<1$. Both vectors were normalized to have unit Euclidean norm. The lower picture shows the discrepancy between the two, and again we see a small contribution that increases periodically away from node 1 .

Theorem 2 makes these observations concrete. The result is strongly motivated by the approach in [22], where exponentially accurate approximations were constructed. We extend this approach in order to obtain an explicit expression for the PerronFrobenius eigenvector. The technique of proof is similar to that in Theorem 1. However, we point out that whereas the Katz parameter $\alpha$ in Theorem 1 is fixed, the Perron-Frobenius eigenvalue $\lambda$ in Theorem 2 is dependent upon $N$.

TheOREM 2. For the undirected ring plus directed shortcut network with adjacency matrix $A=C+E$, let $\lambda$ denote the Perron-Frobenius eigenvalue. Then, for sufficiently large $N$, we have $2<\lambda<5 / 2$ and the Perron-Frobenius eigenvector $x$ has the form

$$
x_{i}=s_{1}^{p(i)}+s_{2}^{p(i)-N},
$$


where $s_{1}, s_{2}$ are the roots of the palindromic quadratic $s^{2}-\lambda s+1$, so that

$$
s_{1}=\frac{\lambda-\sqrt{\lambda^{2}-4}}{2}, \quad s_{2}=\frac{\lambda+\sqrt{\lambda^{2}-4}}{2},
$$

with $s_{2}=1 / s_{1}$ and $0<s_{1}<1<s_{2}$.

Proof. Suppose $N$ is even.

Following the proof of Theorem 1 , we start with the ansatz $x_{i}=b+h s^{p(i)}$ for the eigenvector, and attempt to satisfy the requisite equations for the general node, for node 1 and for node $N / 2+1$.

At a general node $k$, that is, in the $k$ th row of (4), we require

$$
b(2-\lambda)+h s^{p(k)}(1 / s+s-\lambda)=0 .
$$

This may be satisfied by setting $b$ to zero and $s$ to the value $s_{1}$ or $s_{2}$. By linearity, we therefore continue with

$$
x_{i}=h_{1} s_{1}^{p(i)}+h_{2} s_{2}^{p(i)} .
$$

Since the eigenvector is only unique up to a scaling, we set $h_{1}=1$, leaving $h_{2}$ and $\lambda$ as free parameters. The conditions in (4) corresponding to nodes 1 and $N / 2+1$ may be written

$$
\begin{aligned}
\left(2 s_{1}-\lambda+s_{1}^{L-1}\right)+h_{2}\left(2 s_{2}-\lambda+s_{2}^{L-1}\right) & =0, \\
s_{1}^{N / 2}\left(2 s_{2}-\lambda\right)+h_{2} s_{2}^{N / 2}\left(2 s_{1}-\lambda\right) & =0,
\end{aligned}
$$

respectively. Since $s_{1}=1 / s_{2}$, multiplying (28) by a factor of $s_{2}^{N / 2}$ we obtain

$$
h_{2}=s_{2}^{-N}\left(\frac{-\left(2 s_{2}-\lambda\right)}{2 s_{1}-\lambda}\right)=s_{2}^{-N}\left(\frac{-\sqrt{\lambda^{2}-4}}{-\sqrt{\lambda^{2}-4}}\right)=s_{2}^{-N} .
$$

Then writing (27) in terms of the remaining unknown, $\lambda$, we have

$$
F(\lambda)=0
$$

where

$$
\begin{aligned}
F(\lambda)= & -\sqrt{\lambda^{2}-4}\left(1-\left(\frac{\lambda-\sqrt{\lambda^{2}-4}}{2}\right)^{N}\right)+\left(\frac{\lambda-\sqrt{\lambda^{2}-4}}{2}\right)^{L-1} \\
& +\left(\frac{\lambda-\sqrt{\lambda^{2}-4}}{2}\right)^{N-L+1} \cdot
\end{aligned}
$$

It is straightforward to show that $F(2)>0$, whereas at $\lambda=5 / 2$ we have

$$
\begin{aligned}
F(5 / 2) & =-\frac{3}{2}\left(1-2^{-N}\right)+2^{1-L}+2^{L-N+1} \\
& =-\frac{3}{2}+O\left(2^{-\theta N / 2}\right)+O\left(2^{-(1-\theta) N / 2}\right)<0,
\end{aligned}
$$

for large $N$. So the continuous function $F$ changes sign in the interval $(2,5 / 2)$.

We have thus established that the nonsymmetric matrix $C+E$ has an eigenvector of the form (24) with an eigenvalue in $(2,5 / 2)$. We can rule out the possibility of an eigenvector existing that has a larger eigenvalue. This follows from [18, Problem 8.4.P15] (which applies to all nonnegative, irreducible matrices) — because we have constructed an eigenvector with all $x_{i}>0$, it must be a Perron-Frobenius eigenvector.

The case where $N$ is odd can be treated in a similar way. 


\section{Extensions.}

7.1. Multiple Rings. The results from Theorems 1 and 2 give exact characterizations of the centrality vectors, and the presence of both geometrically decaying and geometrically increasing components is not intuitively obvious. However, the qualitative nature of the solution, with most weight given to the node with the highest out-degree and with overall centrality decaying according to periodic distance from this node, is no surprise. In this subsection, we show that the type of analysis developed here can be applied to a more general network where the results are not predictable. To our knowledge, this is the first example to capture analytically (rather than experimentally) a change in node centrality ranking as the Katz parameter is varied. To isolate the key ideas, we have chosen a simple network structure, but we note that the same approach can be applied in more general settings.

We study a network built from three undirected, periodic, nearest neighbor $m$ node rings. Nodes 1 to $m$, nodes $m+1$ to $2 m$ and nodes $2 m+1$ to $3 m$ make up rings one, two and three, respectively. Node 1 is given a long range directed shortcut to node $L$, where $1 \ll L \leq m / 2+1$. Similarly, nodes $m+1$ and $2 m+1$ are given long range directed shortcuts to nodes $m+1+L$ and $2 m+1+L$, respectively. An extra node, with index $N=3 m+1$, is then introduced and connected by an undirected edge to the nodes that have shortcuts. Figure 3 illustrates the case where $m=10$ and $L=5$.

By symmetry, we only need to consider Katz centrality for nodes in the first ring, that is, nodes 1 to $m$, and for the additional node, $N$. We note that node 1 has outdegree four, whereas node $N$ has out-degree three. It follows that for small $\alpha$, node 1 will have the higher Katz centrality. However, the three edges possessed by node $N$ connect to nodes that are themselves well-connected, and can propagate more walks round the network than other nodes on the ring. This suggests that as $\alpha$ is increased, and hence longer walk counts become more relevant, Katz centrality may give more relative weight to node $N$. Our aim is to quantify this effect.

We use the ansatz in Theorem 1, but rather than deriving an exact solution, we focus on the dominant terms. For this network, the Katz system (1) reduces to

$$
\begin{aligned}
x_{j}-\alpha\left(x_{j-1}+x_{j+1}\right) & =1, \quad \text { for } 2 \leq j \leq m, \\
x_{1}-\alpha\left(x_{m}+x_{2}+x_{L}+x_{N}\right) & =1, \\
x_{N}-3 \alpha x_{1} & =1 .
\end{aligned}
$$

Inserting the ansatz (15) we find that, as in the proof of Theorem 1, the general equation (31) is solved with $b=1 /(1-2 \alpha)$ and $t=t_{1}$. Using $x_{L} \approx b$ and solving (32) and (33) for $h$, we arrive at

$$
h \approx \frac{\alpha(2+\alpha)}{(1-2 \alpha)\left(1-2 \alpha t_{1}-3 \alpha^{2}\right)} .
$$

As $\alpha$ increases away from zero, the expression (34) is initially positive and changes sign on crossing a pole where

$$
\alpha=\widehat{\alpha}:=\frac{\sqrt{\sqrt{13}-2}}{3} \approx 0.4224 .
$$

In passing, we therefore conjecture that the adjacency matrix for this network has a spectral radius that approaches $1 / \widehat{\alpha}$ as $N \rightarrow \infty$ and hence the Katz system is valid 


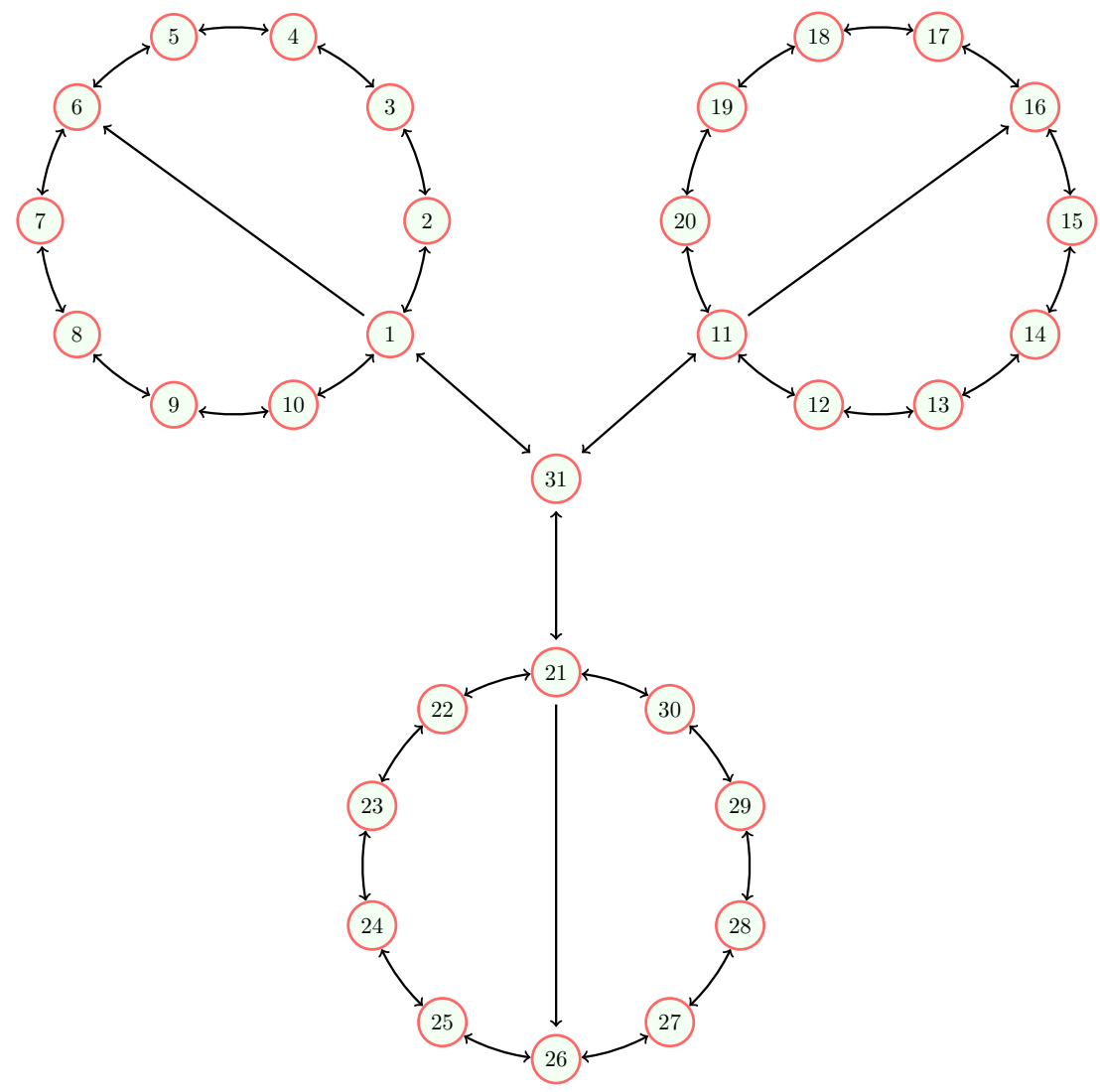

FIG. 3. Illustration of the three-ring undirected network where a directed shortcut is added to each ring, and an extra node is added and given undirected connections to the shortcut nodes. This shows the case $m=10$, so that $N=31$, and $L=6$.

for $0<\alpha<\widehat{\alpha}$. Using $x_{1}=b+h$ and, from (33), $x_{N}=1+3 \alpha x_{1}$, it follows that $x_{N}>x_{1}$ when

$$
\frac{(1-2 \alpha)\left(1-2 \alpha t_{1}-3 \alpha^{2}\right)+(3 \alpha-1)\left(1+2 \alpha\left(1-t_{1}-\alpha\right)\right)}{(1-2 \alpha)\left(1-2 \alpha t_{1}-3 \alpha^{2}\right)}>0 .
$$

For $0<\alpha<\widehat{\alpha}$ the denominator in (35) is positive and the condition reduces to $\alpha>\alpha^{\star}$, where

$$
\alpha^{\star}=\frac{10-\sqrt{13}}{29} \approx 0.2205 .
$$

In summary, we claim that, asymptotically, $\alpha^{\star}$ is the threshold value beyond which node $N$ is regarded as more central than node 1.

Figure 4 supports this conclusion by plotting the ratio $x_{1} / x_{N}$ as $\alpha$ is varied. We used $m=10,000$, so $N=30,001$, with $L=5,001$ and solved the Katz system (1) directly. We see that $\alpha^{\star}$ in (36), marked with a vertical dashed line, predicts the crossover point where $x_{N}$ becomes dominant. It is also interesting to observe that $x_{1} / x_{N}$ is not monotonic in $\alpha$. As further confirmation of the analysis, we found that the approximation $x_{1} / x_{N} \approx(b+h) /(1+3 \alpha(b+h))$ with $h$ in (34) agreed with the 


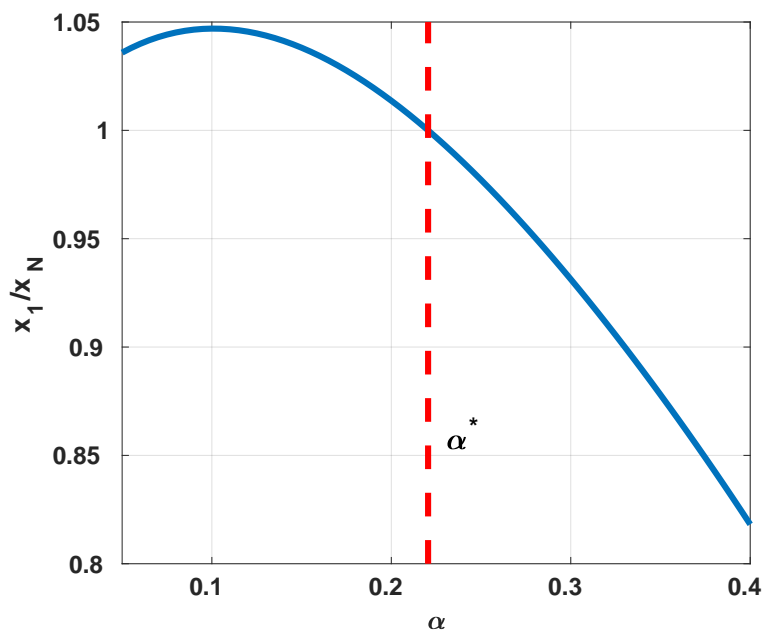

FIG. 4. Ratio of $x_{1}$ to $x_{N}$ for Katz centrality on the $N=30,001$ version of the network in Figure 3. The predicted threshold $\alpha^{\star}$ is marked as a vertical dashed line.

computed values shown in the figure to machine precision. Also, the spectral radius of the adjacency matrix, computed via MATLAB's eigs, matched $1 / \widehat{\alpha}$ to within $10^{-13}$.

7.2. More Neighbors and Shortcuts. Next, we indicate briefly how the analysis in Section 5 may be generalized to rings with more neighbors and shortcuts. Figure 5 shows Katz centrality, with $\alpha=1 / 4.5$, for a network based on a 500 node periodic ring where each node is connected to two clockwise and two counterclockwise neighbors. Three arbitrary, long-range, directed shortcuts were added: $1 \mapsto 200$, $150 \mapsto 390$ and $257 \mapsto 450$. We can see from Figure 5 that nodes 1, 150 and 257 benefit from their extra edge, and the centrality vector has a spike at each of the three locations.

To describe this behavior, consider a general node $k$ that is close to node 1 , but for which $k \neq 1,2, N$. Then the Katz system (1) for this node has the form

$$
x_{k}-\alpha\left(x_{k+1}+x_{k+2}+x_{k-1}+x_{k-2}\right)=1 .
$$

Using the ansatz $x_{i}=b+h r^{p(i)}$, we find that this equation is solved by taking $b=1 /(1-4 \alpha)$ and $r^{2}-\alpha\left(r^{4}+r^{3}+r+1\right)=0$. That palindromic quartic polynomial has four real roots

$$
r_{A} \approx 0.73, \quad r_{B} \approx-0.37, \quad r_{C} \approx 1.37, \quad r_{D} \approx-2.73
$$

As for the three-ring example, we proceed by focusing on the decaying terms, corresponding to roots with absolute value less than one, assuming that the growing terms will be negligible. At nodes 1 and 2 (we could equivalently use 1 and $N$ ), we require

$$
x_{1}-\alpha\left(x_{2}+x_{3}+x_{N}+x_{N-1}+x_{200}\right)=1 \quad \text { and } \quad x_{2}-\alpha\left(x_{3}+x_{4}+x_{1}+x_{N}\right)=1,
$$

respectively. Inserting $x_{200}=b$ and, otherwise, $x_{i}=b+h_{A} r_{A}^{p(i)}+h_{B} r_{B}^{p(i)}$, we obtain the linear system

$$
\left[\begin{array}{cc}
1-\alpha\left(2 r_{A}+2 r_{A}^{2}\right) & 1-\alpha\left(2 r_{B}+2 r_{B}^{2}\right) \\
r_{A}-\alpha\left(1+r_{A}+r_{A}^{2}+r_{A}^{3}\right) & r_{B}-\alpha\left(1+r_{B}+r_{B}^{2}+r_{B}^{3}\right)
\end{array}\right]\left[\begin{array}{c}
h_{A} \\
h_{B}
\end{array}\right]=\left[\begin{array}{c}
b \alpha \\
0
\end{array}\right] .
$$




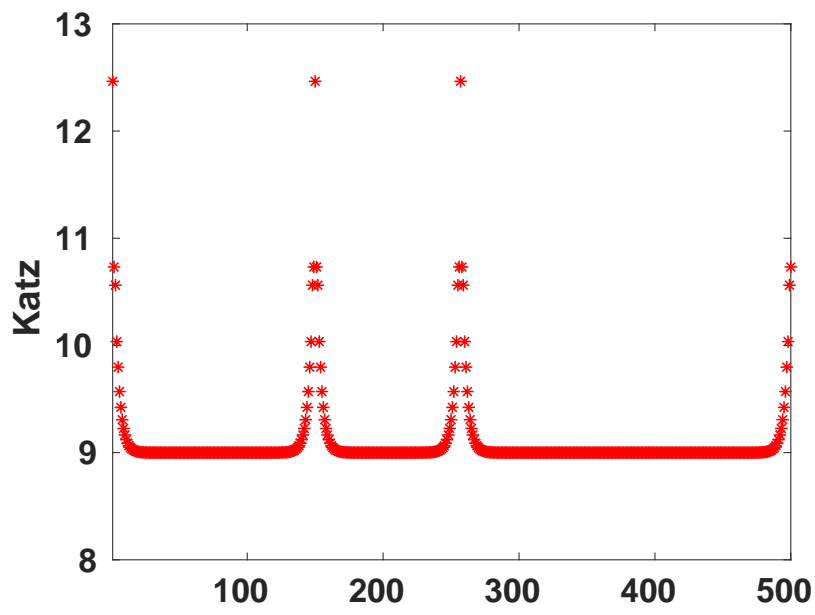

FIG. 5. Components of Katz centrality vector for a 500 node periodic ring with two connections on each side and directed shortcuts added to nodes 1, 150 and 257.

This solves to give $h_{A} \approx 2.73$ and $h_{B} \approx 0.73$.

To cover all the shortcuts, we superimpose three such spikes, so

$$
x_{i} \approx b+h_{A} r_{A}^{p(i)}+h_{B} r_{B}^{p(i)}+h_{A} r_{A}^{p_{150}(i)}+h_{B} r_{B}^{p_{150}(i)}+h_{A} r_{A}^{p_{257}(i)}+h_{B} r_{B}^{p_{257}(i)},
$$

where $p_{L}(i)$ denotes the periodic distance from node $i$ to node $L$; that is

$$
p_{L}(i)=\min (|i-L|,|N-i+L|) .
$$

This approximation agreed with the computed Katz centrality vector to within $2 \times$ $10^{-7}$ in each component. After normalizing both vectors to unit Euclidean norm, the maximum componentwise discrepancy fell below $10^{-9}$.

8. PageRank. We now consider the PageRank system (5) on a periodic ring plus a directed shortcut. To be consistent with the treatment in sections 5 and 6 , we will ensure that node 1 remains the most highly ranked. So the directed shortcut will be added from node $L$ to node 1 . Hence, the adjacency matrix now has the form $A=C+\widehat{E}$, where the rank one matrix $\widehat{E}$ is zero except for $E(L, 1)=1$.

Figure 6 relates to the case where $N=20, L=8$ and $d=0.8$. Asterisks in the upper picture show the components of the PageRank vector $x$ in (5). We see that node 1 is ranked highest, and node $L$, which gives away the extra shortcut, has a low ranking that is slightly higher than its two neighbours. Unlike the cases shown in Figures 1 and 2, the solution does not appear to be periodically symmetric about node 1.

Figure 7 gives a view of the asymptotic $N \rightarrow \infty$ structure by increasing $N$ to 100 , with $L=40$. The solution now appears to be periodically decreasing locally away from node 1 and periodically increasing locally away from node $L$.

Returning to the $N=20$ ring, the circles in the upper picture of Figure 6 show the approximation arising from fitting suitable spikes. The lower picture in Figure 6 

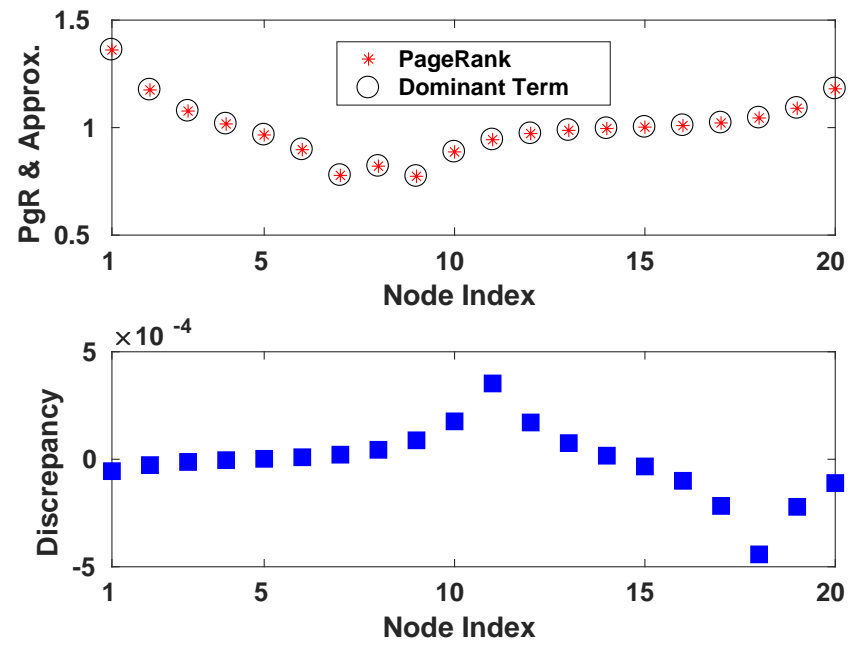

FIG. 6. Upper picture: asterisks show components of the PageRank vector $x$ from (5) and circles show the periodic spike approximation (40)-(41). Lower picture: the discrepancy between these two vectors.

shows the discrepancy, which takes relatively small values that peak and trough at the nodes diametrically opposite 1 and $L$, respectively. We also see in Figures 6 and 7 that node $L$ does not quite fit into the general pattern of periodic growth/decay.

The solutions seen in Figures 6 and 7 may be likened to a positive spike centered at node 1 plus a negative spike centered at node $L$, so we may expect $x_{i}$ to contain a term proportional to $u_{1}^{p(i)}$ and a term proportional to $-u_{1}^{p_{L}(i)}$, where we recall that $p_{L}(i)$ in (37) denotes the periodic distance from node $i$ to node $L$.

An exception occurs at node $L$, which seems to break this pattern. Theorem 3 below shows that the solution does indeed have this general form - plus exponentially small terms - with a simple shift needed at node $L$. The general form (38)-(39) involves five constants $g_{1}, g_{1}^{\prime}, g_{2}, g_{2}^{\prime}, f$ and our proof technique relies on five "special" nodes that impose independent constraints. For this reason, we need to rule out the exceptional case where node $L$ is within one hop of being diametrically opposite node 1 , which would lead to fewer than five special nodes. We therefore assume that the shortcut is chosen so that $0<\theta<1$ in (9). We also treat the PageRank parameter $0<d<1$ as being fixed, independently of $N$.

THEOREM 3. For the undirected ring plus directed shortcut network with adjacency matrix $A=C+\widehat{E}$, for $0<\theta<1$ and for sufficiently large $N$ the unique solution of the PageRank system (5) has the form

$$
x_{i}=1+g_{1} u_{1}^{p(i)}+g_{2} u_{2}^{p(i)}+g_{1}^{\prime} u_{1}^{p_{L}(i)}+g_{2}^{\prime} u_{2}^{p_{L}(i)}, \quad \text { for } i \neq L,
$$

and

$$
x_{L}=1+g_{1} u_{1}^{L-1}+g_{2} u_{2}^{L-1}+g_{1}^{\prime}+g_{2}^{\prime}+f .
$$

Here, $u_{1}, u_{2}, g_{1}, g_{2}, g_{1}^{\prime}, g_{2}^{\prime}, f$ are constants, i.e., independent of $i$, and $u_{1}, u_{2}$ are independent of $N$. In particular, $g_{1}>0, g_{2}>0, g_{1}^{\prime}<0, g_{2}^{\prime}<0, f>0$, and $u_{1}, u_{2}$ are the 


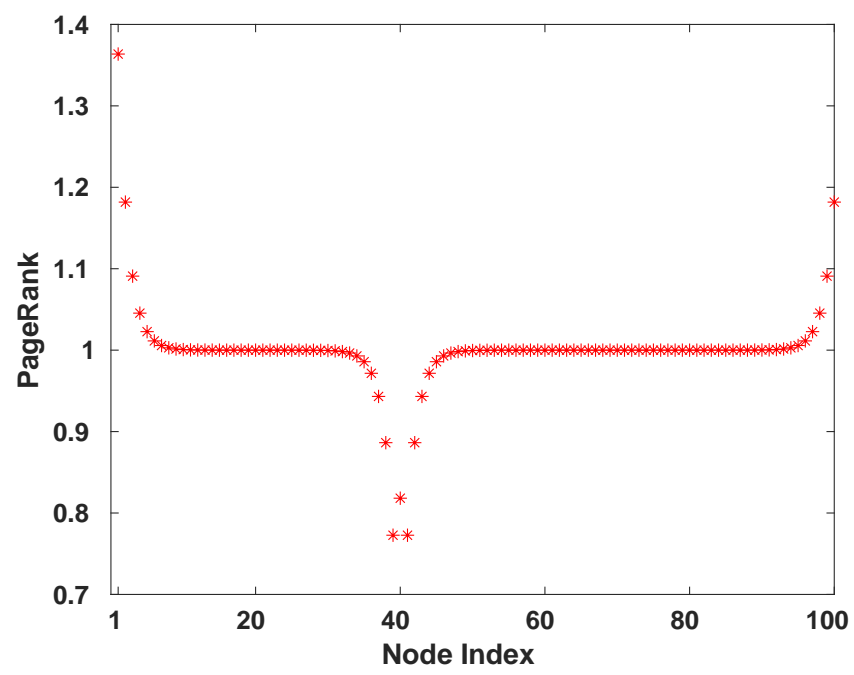

FIG. 7. Asterisks show components of the PageRank vector $x$ from (5) for a larger network: here $N=100$ and $L=40$.

roots of the palindromic quadratic $d u^{2}-2 u+d$, so that

$$
u_{1}=\frac{1-\sqrt{1-d^{2}}}{d}, \quad u_{2}=\frac{1+\sqrt{1-d^{2}}}{d} .
$$

Hence, $u_{2}=1 / u_{1}$ and $0<u_{1}<1<u_{2}$. Moreover, the terms in (38) and (39) involving $g_{2}$ and $g_{2}^{\prime}$ are exponentially small asymptotically, in the sense that

$$
\begin{aligned}
x_{i} & =1+g_{1} u_{1}^{p(i)}+g_{1}^{\prime} u_{1}^{p_{L}(i)}+O\left(u_{1}^{\theta N / 2}\right), \quad \text { for } i \neq L, \\
x_{L} & =1+g_{1}^{\prime}+f+O\left(u_{1}^{\theta N / 2}\right) .
\end{aligned}
$$

We also have

$$
\begin{gathered}
g_{1}=\frac{d}{1+2 \sqrt{1-d^{2}}}+O\left(u_{1}^{\theta N / 2}\right), \\
g_{1}^{\prime}=\frac{-1}{1+2 \sqrt{1-d^{2}}}+O\left(u_{1}^{\theta N / 2}\right), \\
f=\frac{\sqrt{1-d^{2}}}{1+2 \sqrt{1-d^{2}}}+O\left(u_{1}^{\theta N / 2}\right) .
\end{gathered}
$$

Proof. As in the proofs of Theorems 1 and 2, we show by direct substitution that $x$ satisfies the required conditions. Because the style of analysis is similar, we omit some of the fine details.

Assume that $N$ is even.

For a general node $k$, the PageRank system (5) requires $x_{k}-d\left(x_{k-1}+x_{k-1}\right) / 2=$ $1-d$. It is straightforward to verify that (38) satisfies this condition.

It remains to check the system at 
- node 1 , where there are three incoming edges,

- node $L$, where, from (39), the general form of the solution has undergone a shift,

- node $L+1$ (or, equivalently, node $L-1$ ), where the nonstandard node $L$ is involved,

- node $N / 2+1$, which has neighbours that are the same periodic distance from node 1 ,

- node $N / 2+L$, which has neighbours that are the same periodic distance from node $L$.

Our equation for node 1 is $x_{1}-d\left(x_{2} / 2+x_{N} / 2+x_{L} / 3\right)=1-d$. Using (38) and (39), and simplifying, we arrive at

$$
g_{1}\left(1-d\left(u_{1}+\frac{1}{3} u_{1}^{L-1}\right)\right)+g_{2}\left(1-d\left(u_{2}+\frac{1}{3} u_{2}^{L-1}\right)\right)-\frac{1}{3} d\left(g_{1}^{\prime}+g_{2}^{\prime}+f+1\right)=0 .
$$

For node $L$, we have $x_{L}-d\left(x_{L-1} / 2+x_{L+1} / 2\right)=1-d$, which simplifies to

$$
g_{1}^{\prime}\left(1-d u_{1}\right)+g_{2}^{\prime}\left(1-d u_{2}\right)+f=0 .
$$

Node $L+1$ gives $x_{L+1}-d\left(x_{L} / 3+x_{L+2} / 2\right)=1-d$, from which we obtain

$$
g_{1} u_{1}^{L-1}+g_{2} u_{2}^{L-1}+g_{1}^{\prime}+g_{2}^{\prime}-2 f=-1 .
$$

At node $N / 2+1$ we have $x_{N / 2+1}-d\left(x_{N / 2} / 2+x_{N / 2+2} / 2\right)=1-d$, which leads to

$$
g_{2}=g_{1} u_{1}^{N} .
$$

Similarly, at node $N / 2+L$, the condition $x_{N / 2+L}-d\left(x_{N / 2+L-1} / 2+x_{N / 2+L+1} / 2\right)=$ $1-d$ leads to

$$
g_{2}^{\prime}=g_{1}^{\prime} u_{1}^{N} .
$$

Using (48) and (49) to eliminate $g_{2}$ and $g_{2}^{\prime}$, we are left with three linear equations, (45)-(47), for the three unknowns $g_{1}, g_{1}^{\prime}$ and $f$. These may be written in the form

$$
(B+\Delta B) v=r,
$$

where

$$
B=\left[\begin{array}{ccc}
1-d u_{1} & -d / 3 & -d / 3 \\
0 & 1-d u_{1} & 1 \\
0 & 1 & -2
\end{array}\right], \quad v=\left[\begin{array}{c}
g_{1} \\
g_{1}^{\prime} \\
f
\end{array}\right], \quad r=\left[\begin{array}{c}
d / 3 \\
0 \\
-1
\end{array}\right],
$$

and $\Delta B \in \mathbb{R}^{3 \times 3}$ is such that $\|\Delta B\|_{\infty}=O\left(u_{1}^{\theta N / 2}\right)$. Writing $u_{1}$ in terms of $d$, the determinant of $B$ reduces to $-\left(1+2 \sqrt{1-d^{2}}\right) \sqrt{1-d^{2}} \neq 0$, so $B$ is nonsingular.

From (50), we have

$$
\left(I+B^{-1} \Delta B\right) v=B^{-1} r .
$$

The matrix $I+B^{-1} \Delta B$ is invertible for sufficiently large $N$, which establishes that (50) has a unique solution. We conclude that $x$ in (38)-(39) solves the PageRank system (5). Moreover, since $\left(I+B^{-1} \Delta B\right)^{-1}=I+O(\Delta B)$, we see that $v=B^{-1} r+O\left(u_{1}^{\theta N / 2}\right)$, which leads to (42)-(44). The relations (48) and (49) then show that $g_{2}>0$ and $g_{2}^{\prime}<0$ and also give the expansions (40) and (41).

The result for $N$ odd follows similarly. 
For the examples in Figures 6 and 7, we see that the node giving out the extra link, with index $L$, has a slightly larger PageRank value than its immediate neighbors, but not more than its second-neighbors. The following corollary shows that this behavior is generic.

COROllary 1. For the undirected ring plus directed shortcut network with adjacency matrix $A=C+\widehat{E}$, for sufficiently large $N$ the PageRank vector satisfies

$$
\max \left(x_{L+1}, x_{L-1}\right)<x_{L}<\min \left(x_{L+2}, x_{L-2}\right) .
$$

Proof. It follows from Theorem 3 that, ignoring asymptotically small terms,

$$
x_{L}=1+g_{1}^{\prime}+f, \quad x_{L+1}=x_{L-1}=1+g_{1}^{\prime} u_{1}, \quad x_{L+2}=x_{L-2}=1+g_{1}^{\prime} u_{1}^{2} .
$$

Using (42), (43) and (44) we find that

$$
x_{L}-x_{L+1}=x_{L}-x_{L-1}=\frac{(1-d)\left(1-\sqrt{1-d^{2}}\right)}{d\left(1+2 \sqrt{1-d^{2}}\right)}>0,
$$

and

$$
x_{L}-x_{L+2}=x_{L}-x_{L-2} \frac{-\sqrt{1-d^{2}}\left(\sqrt{1-d^{2}}-1\right)^{2}}{d^{2}\left(1+2 \sqrt{1-d^{2}}\right)}<0 .
$$

We can at least partially explain the ordering in Corollary 1 by noting that nodes $L+1$ and $L-1$ suffer because one of their two neighbors, node $L$, has three outgoing links. Hence the "vote of confidence" from node $L$ is split three ways instead of two. As a consequence, node $L$ itself suffers from having two lowly-ranked neighbors. What is perhaps less obvious, but proved in Corollary 1 , is that nodes $L-1$ and $L+1$ can never rise above node $L$, which itself can never rise above nodes $L-2$ and $L+2$.

9. Discussion. In this work, we have addressed issues in matrix analysis concerning modified rings. In particular, we have extended the approximation technique from [22] in order to give exact solutions, and applied these ideas to spectral and linear system problems arising in network science, where the matrix modifications correspond to shortcuts in the classic Watts-Strogatz model. The results give a complete understanding of the structure of the centrality vectors on this class, and in the case of PageRank centrality they reveal an unexpected nonmonotonicty in the rankings. We also showed how the same techniques can be used to study more general networks where the node ranking is not predictable in advance.

There is clearly much potential for further work in this area. An immediate issue is the rigorous treatment of the general $k$-neighbor ring, which would involve an understanding of the roots of the palindromic polynomial $r^{k}-\alpha\left(r^{2 k}+\cdots+r^{k+1}+\right.$ $r^{k-1}+\cdots+1$ ). Furthermore the questions addressed here could be posed on (a) higher dimensional lattices, such as nearest-neighbor connections on a torus, (b) hierarchical "network of network" structures, or (c) non-periodic lattices where boundary effects may be significant. Multiple shortcuts could be included, with various asymptotic scalings for their length and separation. Also, in the spirit of [30], random shortcuts could be analysed in a suitable probabilistic framework.

10. Appendix. In Theorem 2 it was sufficient to have a crude bound on the associated Perron-Frobenius eigenvalue. Here, we derive a sharper result that justifies the restriction $0<\alpha<1 / 2$ imposed in Section 5 . 
Theorem 4. Given any $\epsilon>0$ and $K>0$, for sufficiently large $N$ the PerronFrobenius eigenvalue $\lambda$ in Theorem 2 is such that

$$
2+\frac{K}{N^{2}}<\lambda<2+\frac{K}{N^{2-\epsilon}} .
$$

Proof. We consider the case $N$ even, and investigate the roots of $F(\lambda)$ in (30). If $\lambda$ has the form

$$
\lambda=2+K N^{-\beta}
$$

for some $\beta>0$, then to leading order in (25) we have

$$
\begin{aligned}
& s_{1}=1-K^{1 / 2} N^{-\beta / 2}+O\left(N^{-\beta}\right), \\
& s_{2}=1+K^{1 / 2} N^{-\beta / 2}+O\left(N^{-\beta}\right) .
\end{aligned}
$$

Now suppose $\beta=2$. Recalling that $L$ is proportional to $N$, we have

$$
\begin{aligned}
\log s_{1}^{L-1} & =(L-1) \log \left(1-K^{1 / 2} N^{-1}+O\left(N^{-2}\right)\right) \\
& =(L-1)\left(-K^{1 / 2} N^{-1}\right)+O\left(N^{-1}\right) \\
& \rightarrow-\gamma K^{1 / 2}
\end{aligned}
$$

for some fixed $\gamma$. So

$$
s_{1}^{L-1} \rightarrow e^{-\gamma K^{1 / 2}}
$$

Similarly,

$$
s_{1}^{N-L+1} \rightarrow e^{-(1-\gamma) K^{1 / 2}} \text { and } \quad s_{1}^{N} \rightarrow e^{-K^{1 / 2}} .
$$

Since $F(\lambda)$ in (30) may be written

$$
F(\lambda)=\left(2 s_{1}-\lambda\right)\left(1-s_{1}^{N}\right)+s_{1}^{L-1}+s_{1}^{N-L+1},
$$

we conclude that it takes the form of an asymptotically small negative term plus positive terms that are bounded away from zero. Hence $F(\lambda)>0$ for large $N$.

Now suppose $\beta<2$. Similar analysis shows that, as $N \rightarrow \infty$,

$$
s_{1}^{L-1} \rightarrow 0, \quad s_{1}^{N-L+1} \rightarrow 0, \quad s_{1}^{N} \rightarrow 0,
$$

at rates that are exponential; that is, as fast as $e^{-\nu N^{1-\beta / 2}}$ for some fixed $\nu>0$. It follows that $F(\lambda)$ in (30) is dominated by the term $-2 \sqrt{K} N^{-\beta / 2}$, so $F(\lambda)<0$ for sufficiently large $N$.

The case where $N$ is odd can be treated in a similar manner.

Acknowledgements We thank two reviewers for very detailed feedback on our initial submission.

\section{REFERENCES}

[1] M. Aprahamian, D. J. Higham, and N. J. Higham, Matching exponential-based and resolventbased centrality measures, Journal of Complex Networks, 4 (2016), pp. 157-176, https: //doi.org/10.1093/comnet/cnv016.

[2] S. Aral, Social science: Poked to vote, Nature, 489 (2012), pp. 212-214, https://doi.org/10. 1038/489212a, http://dx.doi.org/10.1038/489212a.

[3] A. Barbour and G. Reinert, Small worlds, Random Structures and Algorithms, 19 (2001), pp. $54-74$. 
[4] M. Benzi And C. KLymko, On the limiting behavior of parameter-dependent network centrality measures, SIAM J. Matrix Anal. Appl., 36 (2015), pp. 686-706.

[5] P. Boldi, A. Luongo, And S. Vigna, Rank monotonicity in centrality measures, tech. report, 2017, http://vigna.di.unimi.it/ftp/papers/rank.pdf.

[6] F. Bonchi, P. Esfandiar, D. F. Gleich, C. Greif, and L. V. Lakshmanan, Fast matrix computations for pairwise and columnwise commute times and Katz scores, Internet Mathematics, 8 (2012), pp. 73-112.

[7] A. Böttcher, M. Embree, And M. Lindner, Spectral approximation of banded Laurent matrices with localized random perturbations, Integral Equations and Operator Theory, 42 (2002), pp. 142-165.

[8] A. Böttcher, M. Embree, And V. I. Sokolov, On large Toeplitz band matrices with an uncertain block, Linear Algebra and its Applications, 366 (2003), pp. 87-97.

[9] A. Böttcher, M. Embree, And V. I. Sokolov, The spectra of large Toeplitz band matrices with a randomly perturbed entry, Math. Comp., 72 (2003), pp. 1329-1348.

[10] M. Catral, M. Neumann, And J. XU, Matrix analysis of a Markov chain small-world model, Linear Algebra and its Applications, 409 (2005), pp. 126-146.

[11] T. P. Chartier, E. Kreutzer, A. N. Langville, and K. E. Pedings, Sensitivity and stability of ranking vectors, SIAM J. Scientific Computing, 33 (2011), pp. 1077-1102.

[12] E. Estrada, The Structure of Complex Networks, Oxford University Press, Oxford, 2011.

[13] L. C. Freeman, Centrality in social networks conceptual clarification, Social Networks, 1 (1978), pp. 215-239.

[14] D. F. Gleich, PageRank beyond the web, SIAM Review, 57 (2015), pp. 321-363, https://doi. org $/ 10.1137 / 140976649$.

[15] P. Grindrod, Mathematical Underpinnings of Analytics, Oxford University Press, Oxford, 2014.

[16] D. J. Higham, A matrix perturbation view of the small world phenomenon, SIAM Journal on Matrix Analysis and Applications, 25 (2003), pp. 429-444.

[17] D. J. Higham, Greedy pathlengths and small world graphs, Linear Algebra and Its Applications, 416 (2006), pp. $745-758$.

[18] R. A. Horn and C. R. Johnson, Matrix Analysis, Cambridge University Press, Cambridge, 2nd ed., 2013.

[19] L. KATZ, A new index derived from sociometric data analysis, Psychometrika, 18 (1953), pp. 39-43.

[20] A. N. Langville and C. D. Meyer, Google's PageRank and Beyond: The Science of Search Engine Rankings, Princeton University Press, Princeton, NJ, USA, 2006.

[21] D. Lazer, A. Pentland, L. Adamic, S. Aral, A.-L. Barabási, D. Brewer, N. Christakis, N. Contractor, J. Fowler, M. Gutmann, and T. Jebara, Computational social science, Science, 323 (2009), pp. 721-723.

[22] X. Liu, G. Strang, AND S. OTT, Localized eigenvectors from widely spaced matrix modifications, SIAM J. Discrete Math., 16 (2003), pp. 479-498.

[23] Z. Lu, B. Savas, W. TAng, And I. Dhillon, Supervised link prediction using multiple sources, in Data Mining (ICDM), 2010 IEEE 10th International Conference on, Dec. 2010, pp. 923 $-928$.

[24] R. Monasson, Diffusion, localization and dispersion relations on "small-world" lattices, Eur. Phys. J. B, 12 (1999), pp. 555-567.

[25] M. E. J. Newman, Networks: an Introduction, Oxford Univerity Press, Oxford, 2010.

[26] M. E. J. Newman, C. Moore, and D. J. Watts, Mean-field solution of the small-world network model, Phy. Rev. Lett., 84 (2000), pp. 3201-3204.

[27] M. E. J. Newman And D. J. WatTs, Renormalization group analysis of the small-world network model, Physics Letters A, 263 (99), pp. 341-346.

[28] L. Page, S. Brin, R. Motwani, and T. Winograd, The PageRank citation ranking: Bringing order to the web, Technical Report, Stanford University, 1998.

[29] S. Wasserman and K. Faust, Social Network Analysis: Methods and Applications, Cambridge University Press, Cambridge, 1994.

[30] D. J. Watts and S. H. Strogatz, Collective dynamics of 'small-world' networks, Nature, 393 (1998), pp. 440-442.

[31] J. XU, Markov chain small-world model with asymmetric transition probabilities, Electronic Journal of Linear Algebra, 17 (2008), pp. 1-22. 